\title{
Structural changes and expression of hepatic fibrosis-related proteins in coculture of Echinococcus multilocularis protoscoleces and human hepatic stellate cells
}

\author{
Deping Cao ${ }^{1 *} \mathbb{D}$, Emad Shamsan ${ }^{2,5^{*}}$, Bofan Jiang ${ }^{3}$, Haining Fan ${ }^{3,4}$, Yaogang Zhang ${ }^{3}$ and
} Mustafa Abdo Saif Dehwah ${ }^{5}$

\begin{abstract}
Background: Echinococcus multilocularis is the causative agent of human hepatic alveolar echinococcosis (AE). AE can cause damage to several organs, primarily the liver, and have severe outcomes, such as hepatic failure and encephalopathy. The main purpose of this study was to explore the interactions between hepatic stellate cells (HSCs) and E. multilocularis protoscoleces (PSCs). The results of this study provide an experimental basis for further examination of the pathogenesis of hepatic fibrosis due to AE infection.

Methods: We investigated the role of Echinococcus multilocularis (Echinococcus genus) PSCs in hepatic fibrosis by examining structural changes and measuring hepatic fibrosis-related protein levels in cocultures of PSCs and human HSCs. Structural changes were detected by transmission electron microscopy (TEM), and levels of the hepatic fibrosisrelated proteins collagen I (Col-I), alpha-smooth muscle actin (a-SMA) and osteopontin (OPN) were measured by western blotting and enzyme-linked immunosorbent assay (ELISA).

Results: Under coculture (1) both PSCs and HSCs exhibited morphological changes, as observed by TEM; (2) Col-I, a-SMA, and OPN expression levels, which were determined by western blotting and ELISA, significantly increased after 3 days of incubation.

Conclusions: The results of this study provide insights into the molecular mechanisms of AE-induced hepatic fibrosis. Keywords: Echinococcus multilocularis, Hepatic stellate cell, Hepatic fibrosis, Protoscoleces, Collagen-I, Alpha-smooth muscle actin, Osteopontin
\end{abstract}

\section{Background}

Alveolar echinococcosis (AE), one of the deadliest human diseases, is caused by Echinococcus multilocularis $[1,2]$ and is prevalent in most of the northern

\footnotetext{
*Correspondence: ghmccdp@163.com; 3248088030@qq.com 1 Department of Human Parasitology, Guilin Medical University, Guilin 541199, Guangxi Zhuang, China

2 Department of Immunology, Faculty of Medicine, Qinghai University, Xining 810001, Qinghai, China

Full list of author information is available at the end of the article
}

hemisphere [3, 4]. Epidemiological surveys have shown that $\mathrm{AE}$ is common in central Asia, including areas of Kyrgyzstan, Kazakhstan, and northwest China [5, 6]. Protoscoleces (PSCs) of E. multilocularis invade the liver and trigger hepatic fibrosis. Hepatic cells at this phase of the disease are likely damaged by toxic products of the metacestodes [7]. Peri-parasite granulomas form in the liver and cause irreversible hepatic fibrosis, which makes surgical resection difficult, and secondary infections typically occur [8]. Labsi et al. $[9,10]$ original author(s) and the source, provide a link to the Creative Commons licence, and indicate if changes were made. The images or other third party material in this article are included in the article's Creative Commons licence, unless indicated otherwise in a credit line to the material. If material is not included in the article's Creative Commons licence and your intended use is not permitted by statutory regulation or exceeds the permitted use, you will need to obtain permission directly from the copyright holder. To view a copy of this licence, visit http://creativecommons.org/licenses/by/4.0/. The Creative Commons Public Domain Dedication waiver (http://creativeco mmons.org/publicdomain/zero/1.0/) applies to the data made available in this article, unless otherwise stated in a credit line to the data. 
found that aqueous extract of Punica granatum peel and IL-17A are good candidate treatments for cystic hydatidosis in humans. Patients with $\mathrm{AE}$ can remain asymptomatic for up to 15 years [11], and $\mathrm{AE}$ is often at a late stage when patients are first seen at clinic. $\mathrm{AE}$ is fatal in $94 \%$ of cases, particular when it has not been diagnosed and the patient treated [12,13]. The oncosphere produced during $\mathrm{AE}$ grows like a tumour, which is why it is also referred to as "worm cancer". It is impossible to completely remove the tumour because of its growth. Therefore, understanding the mechanisms underlying the interactions between the parasite and humans and the pathogenesis of $\mathrm{AE}$ is necessary to develop treatments for echinococcosis-induced liver damage.

Hepatic stellate cells (HSCs), which are the major cells responsible for the formation of extracellular matrix (ECM) proteins during cirrhosis, are found in the space of Disse and act as a major storage site for vitamin A $[14,15]$. They are stimulated in response to certain growth factors, inflammatory stimuli, and, in the case of liver damage, oxidative stress. For example, damaged liver cells, resident phagocytic cells, infiltrating inflammatory cells, aggregated platelets, and Kupffer cells can activate HSCs. Activated HSCs differentiate into muscle fibroblasts, which express alpha-smooth muscle actin ( $\alpha$-SMA) and accelerate the process of cirrhosis. Under the pathological conditions of cirrhosis, HSCs lose their retinoid (vitamin A) and synthesize a large quantity of ECM components, including collagen, proteoglycans and glycosaminoglycans [16-18]. They also proliferate and migrate, and in addition to the ECM components they generate, such as fibronectin, fibrillar collagen and the aforementioned proteoglycans, lead to the formation of septa in the chronically damaged liver [19]. Moreover, an imbalance in the formation of collagen fibres can cause fibrosis [20]. Hepatic parasitic fibrosis caused by $E$. multilocularis PSCs is a host response associated with immune cell infiltration which activates the differentiation of HSCs into fibroblasts [21]. Therefore, identifying the mechanisms underlying AE-induced liver fibrosis may help us to understand the pathogenesis of $\mathrm{AE}$ and develop better treatments for this disease.

In this study, E. multilocularis PSCs were cultured in modified media to investigate their role in liver fibrosis. The HSC-LX2 cell line was cocultured with PSCs, after which liver fibrosis-related proteins collagen I (Col-I), $\alpha$-SMA, and osteopontin (OPN) were detected and their levels measured.

\section{Methods}

Isolation and cultured the E. multilocularis protoscoleces

Mongolian gerbils (males, 1 month old, $30 \mathrm{~g}$ weight) were infected with E. multilocularis PSCs and then killed with $\mathrm{CO}_{2}$ after 6 months. The livers were collected from the dead gerbils and washed with aseptic phosphate-buffered saline (PBS) under sterile conditions. After separating out the AE cysts, they were mashed into small pieces and passed through an aseptic sieve (mesh size $300 \mu \mathrm{m}$ ) to collect the PSCs. The PSCs were washed five times with PBS containing $1000 \mu \mathrm{g} / \mathrm{mL}$ streptomycin and $1000 \mathrm{U} /$ $\mathrm{mL}$ penicillin. The PSCs were then placed in solution at room temperature (RT; $25-30{ }^{\circ} \mathrm{C}$ ) for $5 \mathrm{~min}$ and debris removed. The viability of the PSCs was tested by staining with $0.1 \%$ trypan blue, as dead PSCs were stained blue. Only PSCs showing > 90\% viability were selected for further use [22]. PSCs were cultured in Dulbecco's modified Eagle's medium (DMEM) containing 10\% (volume/ volume) fetal bovine serum (Gibco, Grand Island, NY), $0.45 \%$ (weight/volume) yeast extract, $0.4 \%$ (weight/volume) glucose, $1000 \mu \mathrm{g} / \mathrm{mL}$ streptomycin, and $1000 \mathrm{U} / \mathrm{mL}$ penicillin at $37{ }^{\circ} \mathrm{C}$ in the presence of $5 \% \mathrm{CO}_{2}$.

\section{Coculture HSC-LX2 and PSC}

HSC-LX2 were obtained from the Beijing University of Biological Sciences (Beijing, China). HSCs were preserved in DMEM supplemented with $10 \%$ fetal bovine serum containing $1000 \mathrm{U} / \mathrm{mL}$ penicillin $\mathrm{G}$ and $1000 \mu \mathrm{g} /$ $\mathrm{mL}$ streptomycin. HSCs were plated on $60-\mathrm{mm}$ plates at $1.5 \times 10^{5}$ cells/plate and the plates were then divided into five groups. Three of these groups were exposed to PSCs and the other two were used as the controls (HSCs only and PSCs only). The three groups of HSCs were exposed to PSCs and were cultured at one of the following PSC:HSC ratios: 1:200, 2:200 and 3:200. All of the groups were incubated at $37{ }^{\circ} \mathrm{C}$ in the presence of $5 \%$ $\mathrm{CO}_{2}$ for 24,48 , and $72 \mathrm{~h}$. The harvested HSC-LX2 were divided into two groups. Cells of the first group were centrifuged and then collected to measure the expression of Col-I, $\alpha-S M A$, and OPN, and the coculture supernatant was collected to measure Col-I, $\alpha$-SMA and OPN levels by enzyme-linked immunosorbent assay (ELISA). Cells of the second group were collected to analyse structural changes.

\section{Transmission electron microscopy}

Cultured HSC-LX2 and PSCs were examined by transmission electron microscopy (TEM) to observe changes in cell morphology. PSC specimens for TEM were immersed in fixative (2.5\% glutaraldehyde) after washing them three times with PBS. The HSC specimens were 
washed with PBS and trypsin was added to the cultured cells. The cells were collected and immersed in fixative (2.5\% glutaraldehyde). Thereafter, the specimens were imaged by TEM (HT7700; Hitachi, Japan).

\section{Western blotting}

Proteins were extracted from cultured cell lysates with phosphatase and protease inhibitor cocktails. Protein $(20 \mu \mathrm{g})$ was separated from each sample by $6 \%$ or $10 \%$ sodium dodecyl sulphate polyacrylamide gel electrophoresis and transferred to a polyvinylidene difluoride membrane. The membranes were blocked using $5 \%$ non-fatty milk in PBS containing 0.1\% Tween-20 for $1 \mathrm{~h}$ at RT, and were then incubated with primary antibodies overnight at $4{ }^{\circ} \mathrm{C}$. Subsequently, the membranes were washed with PBS containing $0.1 \%$ Tween- 20 three times and then incubated with secondary antibodies conjugated with horseradish peroxidase for $90 \mathrm{~min}$ at RT. Protein bands were detected using a Fluor-S MultiImager (Bio-Rad, Hercules, CA). The band density was measured using NHI Image software (version 1.53; NIH, Bethesda, MD); $\beta$-actin served as a loading control. The antibodies used in the analysis were anti-Col-I (ab90395), anti- $\alpha$-SMA (ab7817) and anti-OPN (ab8448) (Abcam, Cambridge, UK).

\section{Measurement of Col-I, a-SMA, OPN in the supernatant}

Col-I, $\alpha$-SMA and OPN levels were measured using ELISA according to the manufacturer's instructions (Cloud-Clone, USA). HSC-LX2 were exposed to different ratios of PSCs for 24, 48, 72, and $96 \mathrm{~h}$. Next, the supernatant was collected and centrifuged at $1000 \times g$ for $20 \mathrm{~min}$ and the pellet collected. The supernatant $(100 \mu \mathrm{L})$ was transferred to a new tube, and the levels of Col-I, $\alpha$-SMA and OPN measured. The samples were then prepared and mixed with the standard sample in 96-well plates. The concentrations of Col-I, $\alpha$-SMA and OPN were determined by measuring the optical density at $450 \mathrm{~nm}$ using a spectrophotometer. Total levels of Col-I, $\alpha$-SMA and OPN are expressed as nanograms per millilitre of protein.

\section{Statistical analysis}

Data are presented as the mean \pm SD. Data were assessed using GraphPad Prism 8.0 software (GraphPad, Inc., La Jolla, CA, USA) and one-way analysis of variance followed by Dunnett's various comparisons test.

\section{Results}

\section{HSC-LX2 promoted the growth of PSCs}

PSCs and HSC-LX2 were cocultured in DMEM for 3 days to examine their interaction. The PSCs became highly motile, developed rapidly and evaginated in the presence of HSC-LX2. TEM was used to observe the structural changes on day 3 in the PSCs cocultured with HSC-LX2.
The micrographs showed a slight change in the walls of the PSCs cocultured with HSC-LX2, as the walls of the PSCs cocultured with HSC-LX2, as the microcilia on the walls of E. multilocularis PSCs become shorter and cell structure disappearing and dissolved on day 3 compared with those of the PSC control (Fig. 1a, d, e, f). Vacuoles appeared and the perinuclear space widening and mitochondria swelling in the cellss of PSCs occurred during co-culture on day 4 (Fig. 1b, c).

\section{Structural changes of HSC-LX2 in coculture with PSCs}

HSC-LX2 were activated at PSC:HSC-LX2 ratios of 1:200 and 2:200 for 3 days, but were inhibited at a PSC:HSCLX2 ratio of 3:200. On day 3 of the coculture, structural changes were analysed by TEM. The lipid droplets that were detected by TEM in control HSC-LX2 on day 0 were present until day 3 (Fig. 2a; Fig. 3a). Lipid droplets were not observed in all the cells, but where they were observed in HSC-LX2 exposed to PSCs they had degenerated by day 3 . This indicated that fibrogenesis had occurred (Fig. 2d; Fig. 3b). Furthermore, the lysosomes increasing and mitochondria swelling occurred in HSCs on the day 3 than normal HSC (Fig. 3c). Some changes in the cytoplasm were observed, such as the mitochondrial cristae space widening and the microfilaments increasing in HSC cytoplasm (Fig. 3d, e). The nuclei of HSCs cocultured wth PSCs also exhibited structural changes in comparison with those of normal control HSCs, as they are malformed or irregular, and microvilli on the cell membrane are increasing and the mitochondria are swelling on day 3 and 4 (Fig. 2d, e).

\section{Col-l expression in HSC-LX2 exposed to PSCs}

Col-I plays a significant role in liver fibrosis. Therefore, the expression of Col-I was examined in HSC-LX2 exposed to PSCs. The expression of Col-I at different ratios of PSC:HSC-LX2 (1:200, 2:200, and 3:200) was measured on days 1,2 and 3 by western blotting (WB) (Fig. 4a). The Col-I level in the coculture supernatant was measured by ELISA. At a PSC:HSC-LX2 ratio of $1: 200$, the expression of Col-I had risen dramatically by days 2 (Dunnett's various comparisons test, the following data processing by this statistical method, $q=3.83, p$ $<0.05)$ and $3(q=5.39, p<0.01)$ (Fig. 4b, ANOVA: $\mathrm{F}_{(3,}$ $\left.{ }_{8)}=14.30, p=0.0014\right)$. At a PSC:HSC-LX2 ratio of 2:200, the level of Col-I had significantly increased by days $1(q=3.37, p<0.05), 2(q=10.04, p<0.001)$ and 3 $(q=7.18, p<0.01)$ for the 2:200 ratio (Fig. 4c, ANOVA: $\left.\mathrm{F}_{(3,8)}=38.49, p<0.0001\right)$. At a PSC:HSC-LX2 ratio of 3:200, the elevation of Col-I was higher on day $2(q=$ $5.15, p<0.001)$ than on days $1(q=7.08, p<0.01)$ and 3 $(q=4.66, p<0.05)$ (Fig. $4 \mathrm{~d}$, ANOVA: $\mathrm{F}_{(3,8)}=42.01, p<$ $0.0001)$. Col-I expression levels had increased gradually 

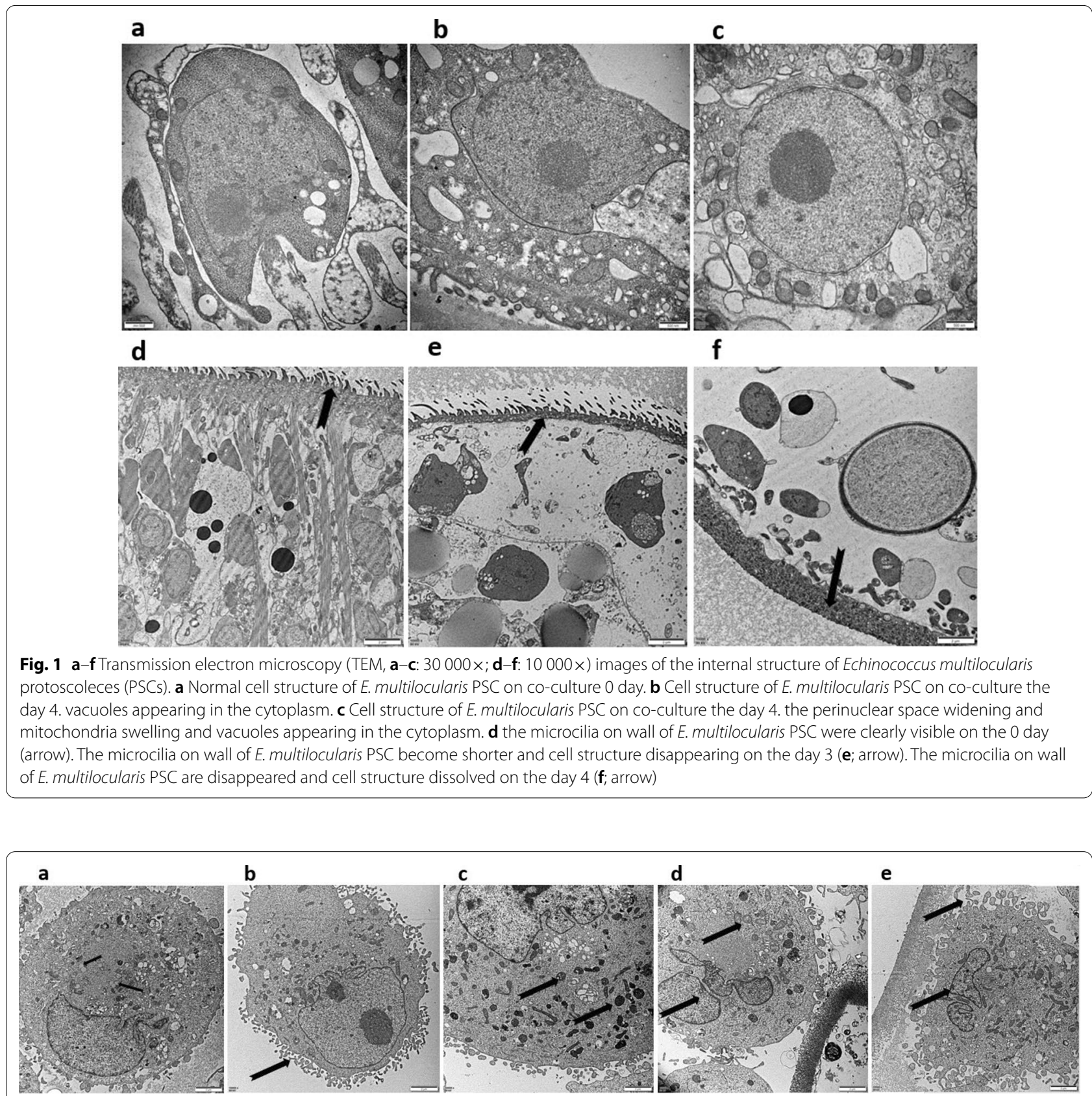

Fig. 2 Structural characteristics of HSCs (TEM, $10000 \times$ ) (HSCs exposed to PSCs). a Lipid droplets are commonly seen in the cytoplasm in HSC on the 0 day (arrow). b Microvilli on the HSC clearly visible on the day 3(arrow). $\mathbf{c}$ Lipid droplets decreasing in cytoplasm; mitochondrial election density increasing and lysosomes increasing in cytoplasm in HSC (arrow). $\mathbf{d}$ The nuclei are malformed and the mitochondria are swelling on the day 3 (arrow). e The nuclei are irregular and microvilli on the cell membrane are increasing on the 4th day

by days 1 and 2 days at PSC:HSC-LX2 ratios of 1:200 and 2:200 (Fig. 4e, ANOVA: $\mathrm{F}_{(10,22)}=34.85, p<0.0001$ ). However, by day 3 , at a ratio of $1: 200$, Col-I had continued to rise, but at ratios of 2:200 and 3:200 showed a decline (Fig. 4f, ANOVA: $\mathrm{F}_{(3,8)}=35.15, p<0.0001$ ).

\section{a-SMA expression in HSC-LX2 exposed to PSCs}

PSCs have been reported to play an essential role in the activation and proliferation of HSCs in coculture accompanied by the expression of $\alpha$-SMA. $\alpha$-SMA expression in HSCs exposed to PSCs was measured by WB (Fig. 5a) and confirmed by ELISA (Fig. 5b-g). $\alpha$-SMA expression increased significantly at a PSC:HSC-LX2 


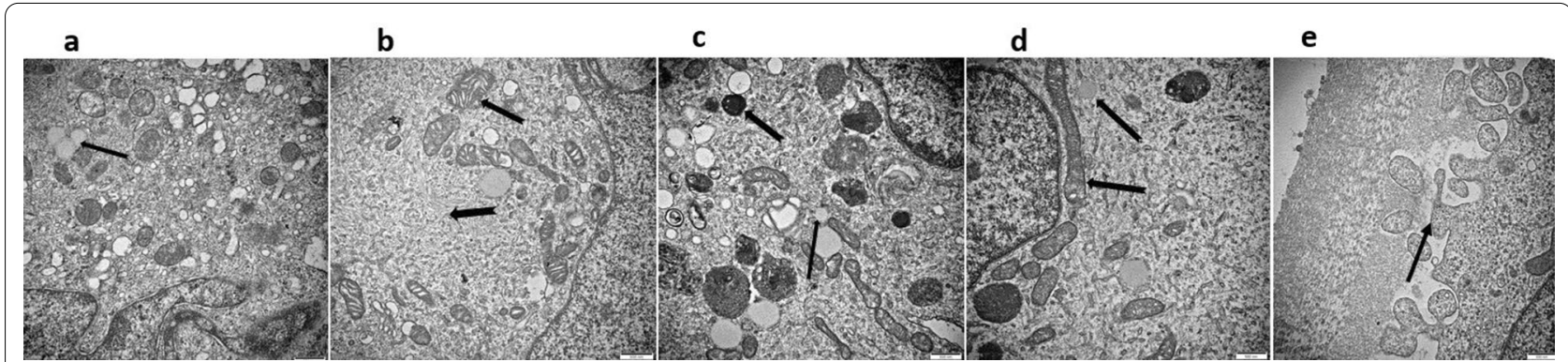

Fig. 3 Structural characteristics of HSC (TEM, 30000 x) (HSCs exposed to PSCs). a The oval lipid droplets clearly visible in HSCS on 0 day. (arrow). b d The mitochondrial cristae space widening and the microfilaments increasing in cytoplasm on the day 3 (arrow). c The lysosomes increasing and mitochondria swelling occurred in HSCs (arrow) on the day 3. e microvilli on the HSC cell membrane (arrow)

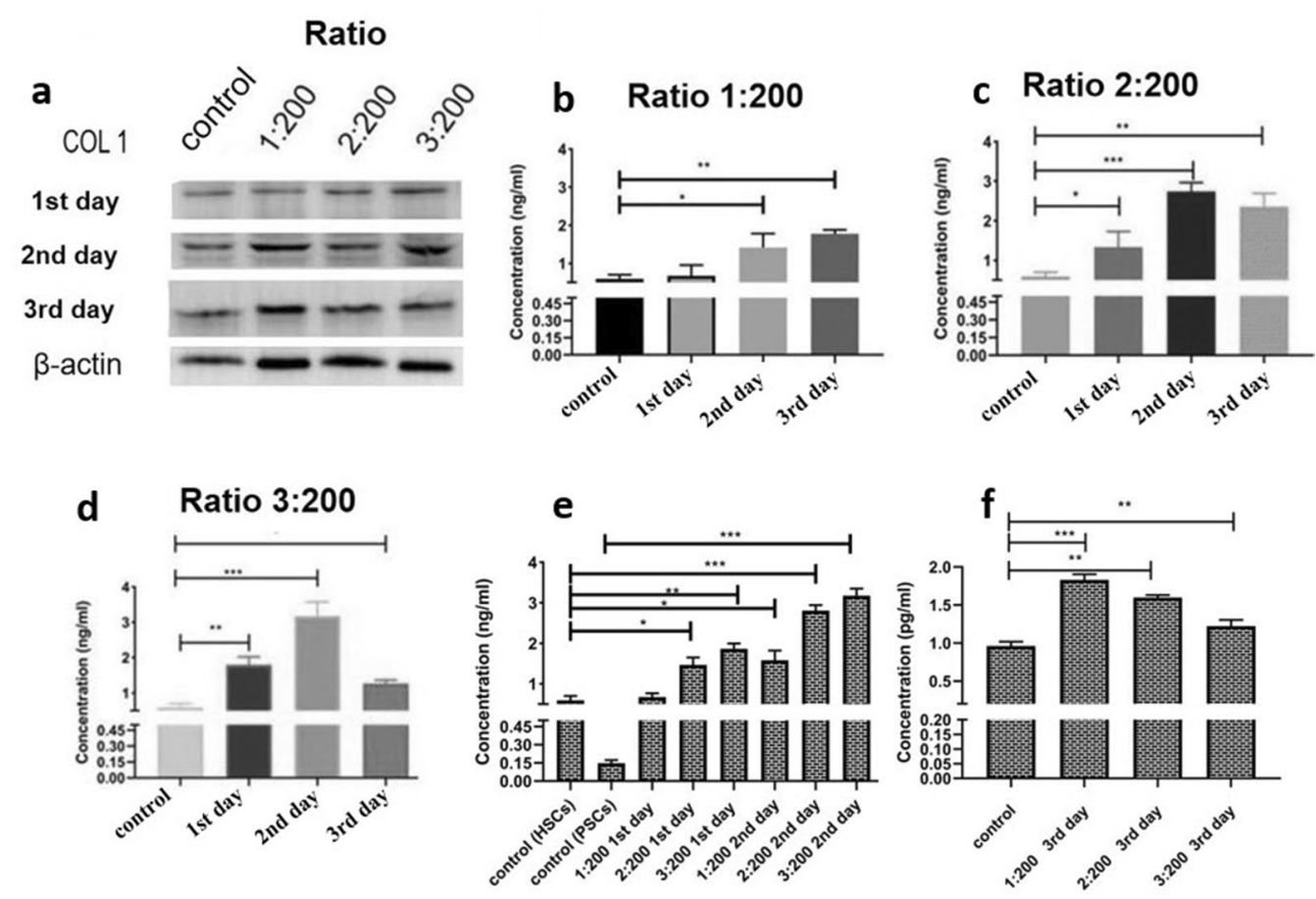

Fig. 4 a-f Collagen I (Col-I) expression profile measured by western blotting (WB) and enzyme-linked immunosorbent assay (ELISA). a Expression of Col-I assessed by WB. b At a PSC:HSC-LX2 ratio of 1:200, Col-I expression had significantly increased by days 2 and 3 but not by day 1 . c Col-I expression at a PSC:HSC-LX2 ratio of 2:200 was significantly higher on days 1, 2 and 3 compared to the control. d At a PSC:HSC-LX2 ratio of 3:200, Col-l expression had increased by days 1 and 2, but decreased by day 3. e Col-l expression increased in HSCs cocultured with PSCs for 48 h. f By day 3, Col-l expression had increased at a PSC:HSC-LX2 ratio of 1:200 but had started to decrease at PSC:HSC-LX2 ratios of 2:200 and 3:200. For other abbreviations, see Fig. 1

ratio of $1: 200$ on the day $2(q=7.54, p<0.01)$ and 3 day $(q=9.69, p<0.01)$ (Fig. 5b, ANOVA: $\mathrm{F}_{(3,8)}=47.12, p<$ $0.0001)$. A significant increase in $\alpha$-SMA expression was observed for the 2:200 ratio on the days 1,2 and 3 ( $q=$ 6.35, $p<0.05 ; q=22.9, p<0.01$; and $q=13.12, p<0.05$, respectively) (Fig. 5c, ANOVA: $\mathrm{F}_{(3,8)}=188.0, p<0.0001$ ). Additionally, a significant elevation in $\alpha$-SMA expression was observed for $3: 200$ on days 1,2 and 3 ( $q=$
8.29, $p<0.01 ; q=16.67, p<0.001$ and $q=12.17, p<0.05$, respectively) (Fig. 5d, ANOVA: $\mathrm{F}_{(3,8)}=119.0, p<0.0001$ ). $\alpha$-SMA expression on day 1 was significantly higher at the PSC:HSC-LX2 ratios of 2:200 and 3:200 but not at 1:200 (Fig. 5e, ANOVA: $\mathrm{F}_{(3,8)}=93.85, p<0.0001$ ). The expression of $\alpha$-SMA on day 2 was significantly higher at a ratio of 2:200 followed by 1:200 and then 3:200 (Fig. 5f, ANOVA: $\left.\mathrm{F}_{(3,8)}=73.37, p<0.0001\right)$. On day $3, \alpha$-SMA 

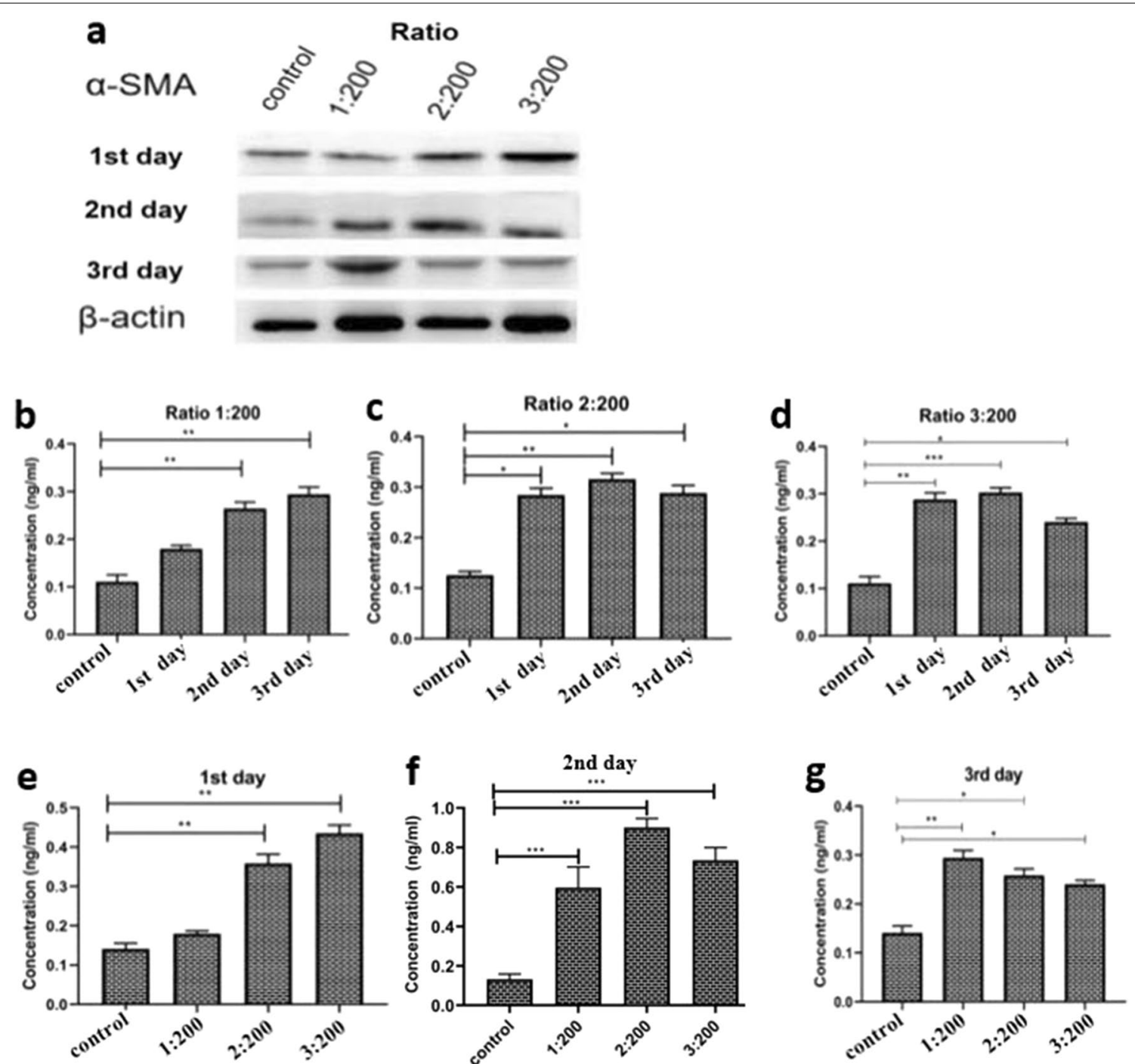

Fig. 5 a-g Alpha-smooth muscle actin (a-SMA) expression profile measured by WB and ELISA. a a-SMA expression assessed by WB. $\mathbf{b}$ a-SMA expression was significantly higher on days 2 and 3 days, but was not on day 1 for the PSC:HSC-LX2 ratio of 1:200. c a-SMA expression was significantly higher on days 1, 2 and 3 for the PSC:HSC-LX2 ratio of 2:200. d For the PSC:HSC-LX2 ratio of 3:200, a-SMA expression had increased by days 1 and 2 but decreased by day 3. e The expression of a-SMA in HSCs cocultured with PSCs for $24 \mathrm{~h}$ increased. f By day 2, a-SMA expression had increased at all ratios of PSC:HSC-LX2 (1:200, 2:200 and 3:200). g By day 3, a-SMA expression had increased at a PSC:HSC-LX2 ratio of 1:200 but had started to decrease at ratios of 2:200 and 3:200. For other abbreviations, see Fig. 1

expression was moderate at a PSC:HSC-LX2 ratio of 1:200 and had decreased at ratios of 2:200 and 3:200 (Fig. 5g, ANOVA: $\mathrm{F}_{(3,8)}=48.42, p<0.0001$ ).

\section{OPN Expression in HSC-LX2 exposed to PSCs}

In addition to Col-I and $\alpha$-SMA, OPN is also a major ECM protein produced during fibrosis. HSCs were incubated under coculture to identify the role of PSCs in OPN expression, which was measured by WB (Fig. 6a). OPN expression increased in the coculture of HSC-LX2 with PSCs during incubation for 4 days (Figs. $6 \mathrm{~b}-\mathrm{d}$ ).
The relationship at a PSC:HSC-LX2 ratio of 1:200 was significant on days $2(q=14.16, p<0.01), 3$ and $4(q=$ 21.78, $p<0.001$ ) (Fig. 6b, ANOVA: $\mathrm{F}_{(4,10)}=116.4, p<$ 0.0001 ); for a ratio of $2: 200$, the results were significant on days $1(q=8.11, p<0.05), 3(q=13.83, p<0.001), 2$ $(q=13.43, p<0.01)$ and $4(q=12.91, p<0.01)$ (Fig. 6 c, ANOVA: $\left.\mathrm{F}_{(4,10)}=68.95, p<0.0001\right)$. At a PSC:HSC-LX2 ratio of 3:200, the relationship was significant on days 1 ( $q$ $=12.29, p<0.01), 2(q=18.20, p<0.001)$ and $3(\mathrm{q}=13.47$, $p<0.001)$ and $4(q=11.48, p<0.001)$ (Fig. 6d, ANOVA: 


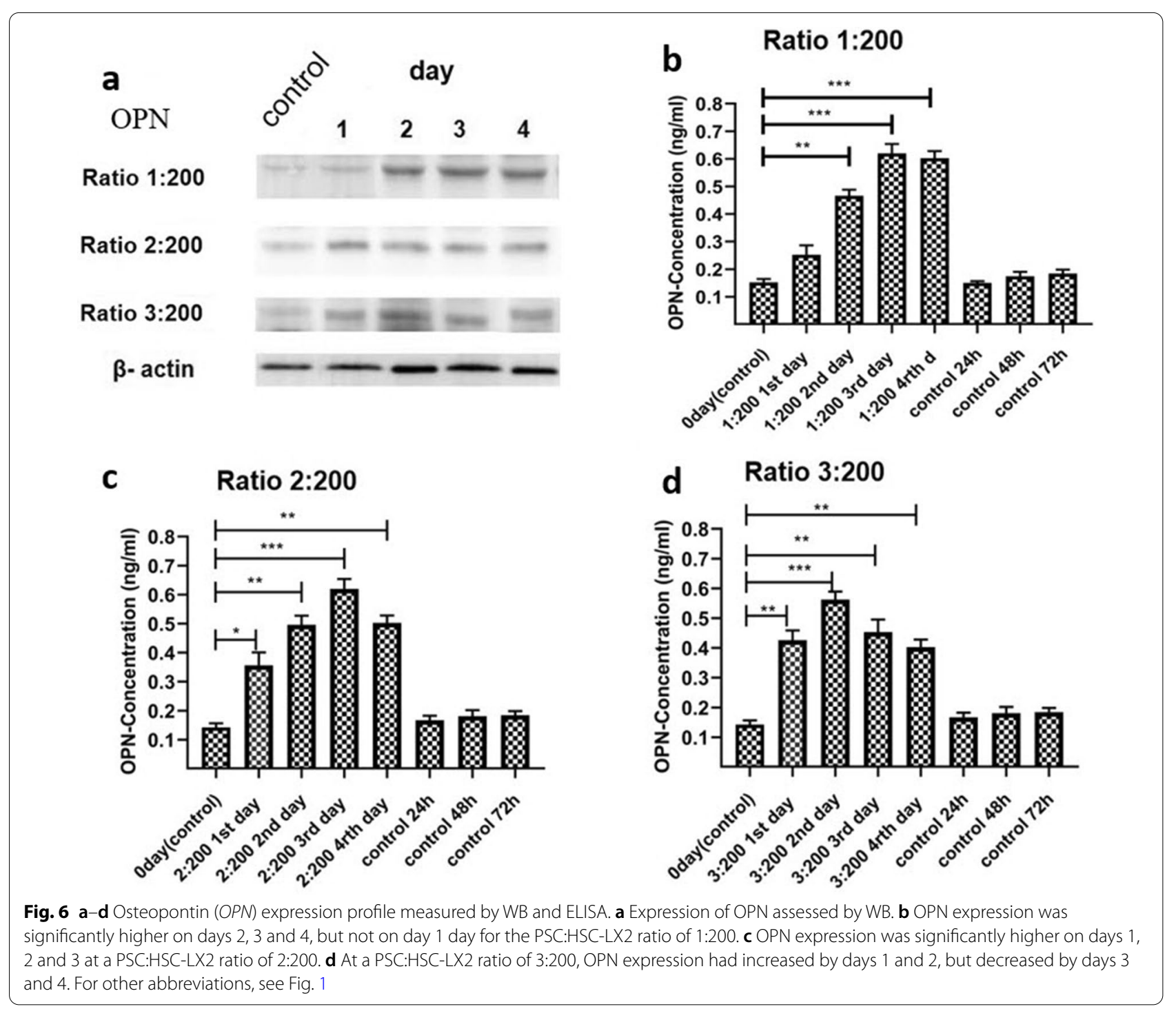

$\left.\mathrm{F}_{(4,10)}=90.42, p<0.0001\right)$; however, OPN expression had increased by days 1 and 2, but decreased by days 3 and 4 .

\section{Discussion}

A cell culture model was designed using E. multilocularis PSC and HSC coculture in vitro to examine the mechanism of liver fibrosis in AE. We evaluated the potential interactions between HSC-LX2 and E. multilocularis PSCs by coculturing them in DMEM. Under coculture, PSCs of E. multilocularis activated HSC-LX2. Li et al. [23] showed that PSCs had rapid motility and were evaginated in the first days of a hepatocyte culture system. In the present study, HSCs exposed to PSCs did not contain lipid droplets, whereas the HSC controls did. The number of lipid-containing HSCs decreased significantly during the transformative phases of liver fibrosis, which indicated that they underwent fibrogenic transformation [24]. All of the features resulting from the exposure of the HSCs to the PSCs, including the division or malformation and irregularity of the nucleus, and the microvilli on the cell membrane increasing and the mitochondria swelling are indicative of apoptosis.

HSCs contain one or one more oval-shaped nucleoli. Morphological changes were observed in the nucleoli of cultured HSC in the form of multiple, thin elongated processes which extended from the cell body [25]. The activation of human HSCs results in the expression of $\alpha$-SMA, which is a specific marker of this process [26]. Our study revealed that the levels of Col-I, $\alpha$-SMA and OPN increased in HSCs when they were exposed to PSCs, and also increased in the supernatant. HSCs exposed to PSCs for $72 \mathrm{~h}$, particularly at a high 
PSC:HSC-LX2 ratio, produced Col-I. This was also found in a study on the effect of Echinococcus spp. PSCs on the process of fibrosis [27]. Additionally, PSCs inhibited the expansion of HSCs by directly targeting TGF- $\beta$ RI/ II [28]. The fluid of the cysts that develop during cystic echinococcosis can inhibit the proliferation of HSCs and increase the levels of the main markers of HSCs, including Col-I and $\alpha$-SMA [27]. Our study showed that PSCs stimulated HSC to secrete Col-I, $\alpha$-SMA, and OPN. HSCs are typically dormant but develop into fibroblasts which accumulate in the ECM when the cells are activated during liver injury [8]. Thus, activation of HSCs is an important process in fibrosis. Collagen synthesis and $\alpha$-SMA levels increased after different incubation periods of HSCs $[8,16]$. A recent study reported that OPN can directly induce the activation and proliferation of HSCs, and that its expression may be either TGF- $\beta$ dependent or non-dependent [29]. Our experiments confirmed that OPN expression increased in HSCs exposed to PSCs. The results of the present study and other studies suggest that HSCs can secrete OPN and that OPN secreted by other cells also activates HSCs.

Many studies have demonstrated the role of HSCs in the synthesis of ECM components, fibrosis and cirrhosis in bovine livers infected with helminths, e.g. Fasciola hepatica and Dicrocoelium dendriticum. HSCs play an important role in the development of fibrosis and other stages of cirrhosis $[24,30]$. TGF- $\beta$ plays a role in the differentiation of HSCs; it is the main factor that activates these cells, which leads to collagen deposition [31]. Fibrosis is a common, progressive pathological process that occurs after extensive liver injury. ECM deposits are characteristic features of liver fibrosis observed after HSC activation. During liver fibrosis, HSCs differentiate and become the main producers of components of the ECM [29,32].

In their study of an in vitro model of liver fibrosis, Ren et al. found that $E$. multilocularis cyst fluid has a significant impact on messenger RNA levels of ERK1/2, JNK1/2 and p38 in rat HSCs, and also observed morphological changes in the HSCs, and karyopyknosis [33]. Gao et al. found that $E$. multilocularis infections could promote the proliferation of HSCs and cause an increase in Col-I and $\alpha$-SMA levels in mice sera, and induce the deposition of collagen fibres in mice livers [34]. Wang et al. successfully cultured E. multilocularis PSCs from jirds to the larval stage, which produced extracellular vesicles in modified RPMI 1640 medium in vitro; the results of that work provided helpful insights for future research on the relationship between $E$. multilocularis PSCs and HSCs in vitro [35].

The findings discussed here show that the infective stage of E. multilocularis is a critical factor in the development of liver fibrosis in AE. The results of our study provide an experimental basis for further elucidation of the pathogenesis of liver fibrosis in $\mathrm{AE}$.

\section{Conclusions}

We explored the interaction between HSCs and E. multilocularis PSCs, and showed that the latter induced the activation of the HSCs, which then produced proteins that play a role in hepatic fibrosis (i.e. Col-I, $\alpha$-SMA, and OPN). These findings may contribute to a better understanding of the pathogenesis of hepatic fibrosis in $\mathrm{AE}$. Additional studies are needed that focus on the role of the PSCs of E. multilocularis in this complicated process to further understand the interaction between this parasite and its host.

\section{Abbreviations}

AE: Alveolar echinococcosis; a-SMA: Alpha-smooth muscle actin; Col-1: Collagen-l; DMEM: Dulbecco's modified Eagle's medium; ECM: Extracellular matrix; ELISA: Enzyme-linked immunosorbent assay; HSCs: Hepatic stellate cells; OPN: Osteopontin; PSC: Protoscoleces; PVDF: Polyvinylidene difluoride; RT: Room temperature; SDS-PAGE: Sodium dodecyl sulfate polyacrylamide gel electrophoresis; TEM: Transmission electron microscopy; WB: Western blotting.

\section{Acknowledgements}

We would like to thank the Key Echinococcosis Laboratory of the Qinghai University Affiliated Hospital for providing the experimental site.

\section{Authors' contributions \\ CDP oversaw the laboratory work, conceived of and designed the study, and drafted the manuscript. ES performed the laboratory work. JBF and ZYG assisted with the laboratory work. FHN revised the draft manuscript and constructed the gerbil models together with AE. MASD provided writing assistance. All authors read and approved the final manuscript.}

\section{Funding}

This work was supported by the Natural Science Foundation of Guangxi Zhuang Autonomous Region (grant 2020GXNSFAA297216) and Qinghai Science and Technology Department Project (grant 2020-ZJ-Y01).

\section{Availability of data and materials}

The datasets used in this study are available from the corresponding author on reasonable request.

\section{Declarations}

Ethics approval and consent to participate

This study was approved by the Ethics Review Board of the Affiliated Hospital of Qinghai University (approval number P-SL-2019054).

Consent for publication

Not applicable.

\section{Competing interests}

The authors declare that they have no competing interests.

\section{Author details}

'Department of Human Parasitology, Guilin Medical University, Guilin 541199, Guangxi Zhuang, China. ${ }^{2}$ Department of Immunology, Faculty of Medicine, Qinghai University, Xining 810001, Qinghai, China. ${ }^{3}$ The Key Echinococcosis Laboratory, Qinghai University Affiliated Hospital, Xining 810001, Qinghai, China. ${ }^{4}$ Department of Hepatobiliary and Pancreatic Surgery, Qinghai University Affiliated Hospital, Xining 810001, Qinghai, China. ${ }^{5}$ Laboratories Department, Faculty of Medical Sciences, Taiz University, Turba Branch, 70270 Taiz, Yemen. 
Received: 12 March 2021 Accepted: 24 September 2021

Published online: 02 December 2021

\section{References}

1. Gottstein B, Wang JH, Blagosklonov O, et al. Echinococcus metacestode: in search of viability marker. Parasite. 2014;21:63.

2. Brehm K, Koziol U. Echinococcus-host interactions at cellular and molecular levels. Adv Parasitol. 2017;95:147-212.

3. Craig PS, McManus DP, Lightowlers MW, et al. Prevention and control of cystic echinococcosis. Lancet Infect Dis. 2007;7:385-94.

4. Brunetti E, Kern P, Vuitton DA. Expert consensus for the diagnosis and treatment of cystic and alveolar echinococcosis in humans. Acta Trop. 2010;114:1-16.

5. Hemphill A, Stadelmann B, Scholl S, et al. Echinococcus metacestodes as laboratory models for the screening of drugs against cestodes and trematodes. Parasitology. 2010;137:569-87.

6. Zhang W, Zhang Z, Wu W, et al. Epidemiology and control of echinococcosis in central Asia, with particular reference to the People's Republic of China. Acta Trop. 2015;141:235-43.

7. Ritler D, Rufener R, Li JV. In vitro metabolomic footprint of the Echinococcus multilocularis metacestode. Sci Rep. 2019;9:19438-50.

8. Gottstein B, Hemphill A. Immunopathology of echinococcosis. Chem Immunol. 1997;66:177-208.

9. Labsi M, Khelifi L, Mezioug D, et al. Antihydatic and immunomodulatory effects of Punica granatum peel aqueous extract in a murine model of echinococcosis. Asian Pac J Trop Med. 2016;3:208-16.

10. Labsi M, Souflia I, Khelif L, et al. In vivo treatment with IL-17A attenuates hydatid cyst growth and liver fibrogenesis in an experimental model of echinococcosis. Acta Trop. 2018:18:6-10.

11. McManus DP, Zhang W, Li J, Bartley PB. Echinococcosis. Lancet. 2003:362:1295-304

12. Moro P, Schantz PM. Echinococcosis: a review. Int J Infect Dis. 2009;13:125-33.

13. Lu Y, Huang Q, Zhou Z, Lu Y. An overview of parasitic diseases by analyzing publications at National Institute of Parasitic Diseases Control and Prevention in the last five years. Chin J Dis Control Prev. 2015;19:86-90.

14. Friedman SL. Hepatic stellate cells: protean, multifunctional, and enigmatic cells of the liver. Physiol Rev. 2008;88:125-72.

15. Galler K, Requardt RP, Glaser U, et al. Single cell analysis in native tissue: quantification of the retinoid content of hepatic stellate cells. Sci Rep. 2016;6:24155

16. Zhang CY, Yuan WG, He P, et al. Liver fibrosis and hepatic stellate cells: etiology, pathological hallmarks and therapeutic targets. World J Gastroenterol. 2016:22:10512-22.

17. Senoo H, Sato M, Imai K. Hepatic stellate cells-from the viewpoint of retinoid handling and function of the extracellar matrix. Kaibogaku Zasshi. J Anat. 1997;72:79-94.

18. Weishkirchen R, Weimer J, Meurer SK, et al. Genetic characteristucs of the human hepatic stellate cell line LX2. PLoS One. 2013;8:e75692.

19. Förster S, Koziol U, Schäfer T, Duvoisin R, Cailliau K, Vanderstraete M, et al The role of fibroblast growth factor signalling in Echinococcus multilocularis development and host-parasite interaction. PLoS Negl Trop Dis. 2019;13:e0006959.

20. Mohammed A, Allen J, Rogan M. Echinococcus granulosus cyst fluid enhances epithelial-mesenchymal transition. Parasite Immunol. 2018;40:e12533.

21. Wang J, Zhang C, Wei X, Blagosklonov O, Lv G, Lu X, et al. TGF- $\beta$ and TGF- $\beta /$ Smad signaling in the interactions between Echinococcus multilocularis and its hosts. PLoS ONE. 2013;8:e55379.

22. Moazeni M, Saharkhiz MJ, Hosseini AA. In vitro lethal effect of ajowan (Trachyspermum ammi L.) essential oil on hydatid cyst protoscoleces. Vet Parasitol. 2012;187:203-8

23. Li L, Chen B, Yan H, Zhao Y, Lou Z, Li J, et al. Three-dimensional hepatocyte culture system for the study of Echinococcus multilocularis larval development. PLoS Negl Trop Dis. 2018;12:e0006309.

24. Nepomnyashchikh Gl, Aidagulova SV, Nepomnyashchikh DL, et al. Ultrastructural and immunohistochemical study of hepatic stellate cells over the course of infectious viral fibrosis and cirrhosis of the liver. B Exp Biol Med. 2006;142:723-8.
25. Herrmann J, Axel M, Gressner AM, et al. Immortal hepatic stellate cells lines: useful tools to study hepatic stellate cell biology and function? J Cell Mol Med. 2007:11:704-22.

26. Chang KT, Tsai MJ, Cheng YT, et al. Comparative atomic force and scanning electron microscopy: an investigation of structural differentiation of hepatic stellate cells. J Struct Biol. 2009;167:200-8.

27. Niu F, Chong S, Qin M, et al. Mechanism of fibrosis induced by Echinococcus spp. Diseases. 2019;7:51.

28. Kwiecinski M, Noetel A, Elfimova N, et al. Hepatocyte growth factor (HGF) inhibits collagen I and IV synthesis in hepatic stellate cells by miRNA-29 induction. PLoS ONE. 2011;6:e24568.

29. Xiao X, Gang Y, Gu Y, et al. Osteopontin contributes to TGF $\beta 1$ mediated hepatic stellate cell activation. Dig Dis Sci. 2012;57:2883-91.

30. Boldin MP, Baltimore D. MicroRNAs, new effectors and regulators of NF-KB. Immunol Rev. 2012;246:205-20.

31. Xu FY, Liu CG, Zhou DD, et al. TGF-B/SMAD pathway and its regulation in hepatic fibrosis. J Histochem Cytochem. 2016;64:157-67.

32. Bartel DP. MicroRNAs: target recognition and regulatory functions. Cell. 2009;136:215-33.

33. Ren $B$, Fang HN, Deng $Y$, et al. Effect of Echinococcus multilocularis cyst fluid on the expression of five MAPK-pathway genes of rat hepatic stellate cells. Zhongguo Ji Sheng Chong Xue Yu Ji Sheng Chong Bing Za Zhi. 2015;33:114-7.

34. Gao HJ, Pang HS, Sun XD, et al. Effects of persistent Echinococcus multilocularis infections on hepatic fibrosis in mice. Zhongguo Xue Xi Chong Bing Fang Zhi Za Zhi. 2021;33:54-61.

35. Wang H, Li J, Guo BP, et al. In vitro culture of Echinococcus multilocularis producing protoscoleces and mouse infection with the cultured vesicles. Parasit Vectors. 2016:9:411.

\section{Publisher's Note}

Springer Nature remains neutral with regard to jurisdictional claims in published maps and institutional affiliations.

Ready to submit your research? Choose BMC and benefit from

- fast, convenient online submission

- thorough peer review by experienced researchers in your field

- rapid publication on acceptance

- support for research data, including large and complex data types

- gold Open Access which fosters wider collaboration and increased citations

- maximum visibility for your research: over 100M website views per year

At BMC, research is always in progress.

Learn more biomedcentral.com/submissions 